\title{
Generalized Solutions of Differential-Operator Equations with Singular White Noise
}

\author{
I. V. Melnikova \\ Institute of Mathematics and Computer Science, Ural Federal University, \\ Yekaterinburg, Russia
}

Received April 28, 2011

\begin{abstract}
In various distribution spaces, we study the Cauchy problem for the equation $u^{\prime}(t)=A u(t)+B \mathbb{W}(t), t \geq 0$, with a singular white noise $\mathbb{W}$ and an operator $A$ generating various regularized semigroups in a Hilbert space. Depending on the properties of the operator $A$, we construct solutions generalized separately and jointly with respect to the time, random, and "space" variables.
\end{abstract}

DOI: $10.1134 /$ S0012266113040083

\section{INTRODUCTION}

Models of various evolution processes considered with regard for random perturbations lead to the Cauchy problem for differential-operator equations with an inhomogeneity in the form of white noise. Of them, fundamental is the Cauchy problem for the first-order equation with an operator $A$ that is the generator of a semigroup of the class $C_{0}$,

$$
X^{\prime}(t)=A X(t)+B \mathbb{W}(t), \quad t \in[0, \tau), \quad \tau \leq \infty, \quad X(0)=\zeta,
$$

which, owing to the irregularity of the white noise $\mathbb{W}$, is reduced by some Wiener process to the problem with an "antiderivative" of $\mathbb{W}$; to this end, it is represented in integral form (e.g., see [1-4]). Therefore, the investigation of problem (1) can be reduced to the analysis of a (not necessarily equivalent) integral problem.

The present paper deals with the construction of generalized solutions of the stochastic Cauchy problem (1), where $A$ is the generator of some regularized semigroup in the Hilbert space $H$, $\{\mathbb{W}(t), t \geq 0\}$ is an $\mathbb{H}$-valued generalized white noise process whose rigorous definition in various distribution spaces will be given below, and $B \in \mathcal{L}(\mathbb{H}, H)$.

Under the above-mentioned conditions, problem (1) is ill-posed for two causes. First, owing to the properties of the operator $A$ generating not a semigroup of the class $C_{0}$ but only some regularized semigroup, in particular, an integrated semigroup or an $R$-semigroup. For such $A$, the operators of solution of the corresponding homogeneous problem $U(t), t \in[0, \tau)$, are not bounded; therefore, instead of the family $\{U(t)\}$ required for the solution of problem (1), one should either construct some regularized family $V=\{V(t), t \in[0, \tau)\}$ or consider operators of solution in spaces of distributions, distributions with respect to the variable $t$ if $V$ is an integrated semigroup or distributions with respect to the "space" variable (defined by the operator $A$ ) if $V$ is an $R$-semigroup [5, pp. 16-25; 6, pp. 34-79]. Second, owing to the irregular behavior of the white noise, in particular, the absence of continuity caused by the independence of the random variables $\mathbb{W}\left(t_{1}\right)=\mathbb{W}\left(t_{1}, \omega\right)$ and $\mathbb{W}\left(t_{2}\right)=\mathbb{W}\left(t_{2}, \omega\right), \omega \in \Omega$, for $t_{1} \neq t_{2}$. The presence of the noise in the equation leads to two distinct approaches to the solution of the stochastic problem (1): (i) the solution of the "differential" problem (1) is replaced by the solution of the above-mentioned integral problem with an "antiderivative" of the white noise, which is a Wiener process defined as

a generalization of Brownian motion; (ii) one solves problem (1) with the white noise $\mathbb{W}$ equal to a generalized derivative of a Wiener process in some distribution space. 
In the present paper, the solution of the posed problem is related to the second approach, whose important components are given by the construction of distribution spaces such that the definition of white noise is possible and the construction of distribution spaces in which the operators of solution of the homogeneous problem, which do not necessarily generate a semigroup of operators of the class $C_{0}$, are defined. The below-suggested idea of definition of a jointly generalized solution permits one to construct distribution spaces in which both above-mentioned difficulties (the absence of a semigroup of operators of the class $C_{0}$ and the irregularity of the white noise) can be overcome. The investigation of the above-posed general problem was started in [7-9], and the investigation dealing with the construction of distributions with respect to several variables was initiated in [10].

In Section 1, we give necessary definitions from the theory of regularized semigroups, abstract Wiener processes, abstract (Hilbert-valued) Schwartz distributions, and stochastic distributions. In Section 2, we construct a solution generalized with respect to time and random variables for problem (1) with generators of integrated semigroups and with an inhomogeneity in the form of white noise. To construct such a solution, we combine the technique of abstract distributions [6, pp. 121-137; 11] providing the construction of semigroups-distributions and the technique of stochastic distributions providing the construction of singular white noise $[7,12]$. In Section 3 , we construct a solution of problem (1) with a white noise and with generators of $R$-semigroups, which is generalized with respect to the random variable and the variable of the space $H$. The desired solution is constructed with the use of stochastic distributions in combination with generalized Ivanov functions [5, pp. 150-153]. Special attention is paid to problems with generators of semigroups with singularities of order $\alpha$ at zero.

A solution generalized with respect to the time variable $t$, the random variable $\omega$, and the space variable is defined as a continuous mapping of the space of test functions depending on $t, \omega$, and the variable of the space $H$, respectively, into an appropriate space of transforms, which is defined for each of solutions. All solutions constructed in Sections 2 and 3 are represented as two terms, the first of which reflects the influence of initial conditions, and the other (a generalized stochastic convolution) represents the influence of random perturbations.

\section{REQUIRED DEFINITIONS: REGULARIZED SEMIGROUPS, ABSTRACT WIENER PROCESSES, ABSTRACT DISTRIBUTIONS}

\subsection{Regularized Semigroups}

Let $A$ be a closed linear operator, and let $R(t), t \geq 0$, be bounded linear operators in a Banach space $H$.

Definition 1. A strongly continuous (with respect to $t$ ) family of bounded operators $\{V(t)$, $t \in[0, \tau)\}=V, \tau \leq \infty$, in $H$ is referred to as an $R$-regularized semigroup with generator $A$ if

$$
V(t) A \zeta=A V(t) \zeta, \quad \zeta \in \operatorname{dom} A, \quad V(t) \zeta=A \int_{0}^{t} V(s) \zeta d s+R(t) \zeta, \quad \zeta \in H .
$$

A semigroup is said to be exponentially bounded if $\|V(t)\| \leq M e^{\varpi t}, t \geq 0$, for some $M>0$ and $\varpi \in \mathbb{R}$ and to be local if $\tau<\infty$.

If $R(t)=\left(t^{n} / n !\right) I$, then the family $V$ is an $n$ times integrated semigroup. If $\overline{\operatorname{dom} A}=H$ and $R(t) \equiv R$ is an invertible bounded operator with dense range, then $V$ is an $R$-semigroup. If $R=I$, then an $R$-semigroup is a semigroup of the class $C_{0}$.

Note that an $R$-semigroup was defined in [13] as a strongly continuous family of bounded operators satisfying the $R$-semigroup relation $V(t+s) R=V(t) V(s), s, t, s+t \in[0, \tau), V(0)=R$, with infinitesimal generator

$$
\mathcal{G} f:=\left(\lambda-L_{\lambda}^{-1}\right) f, \quad \lambda>\varpi, \quad \operatorname{dom} \mathcal{G}=\left\{f \in H: R f \in \operatorname{ran} L_{\lambda}\right\}, \quad L_{\lambda} f:=\int_{0}^{\infty} e^{\lambda t} V(t) f d t
$$

and such a family is referred to as a $C$-semigroup. We prefer the notion of $R$-semigroup in view of its regularizing property and unlike semigroups of the class $C_{0}\left(C_{0^{-}}\right.$-semigroups). 
Integrated semigroups were introduced in [14] via the corresponding "semigroup" relation with an infinitesimal generator, but we use the general definition 1. Examples of integrated, convolution semigroups, and $R$-semigroups and their generators including important differential ones can be found, e.g., in [6, pp. 50-53, 70-79; 15].

\subsection{Wiener Processes}

Let $(\Omega, \mathcal{F}, P)$ be a probability space, let $\mathbb{H}$ be a Hilbert space, and let $Q$ be a linear symmetric nonnegative trace operator with a system of eigenvectors $\left\{e_{i}\right\}$ that forms a basis in the space $\mathbb{H}$ and satisfies the conditions $Q e_{i}=\sigma_{i}^{2} e_{i}$ and $\sum_{i=1}^{\infty} \sigma_{i}^{2}<\infty$.

Definition 2. An $\mathbb{H}$-valued stochastic process $W_{Q}=\left\{W_{Q}(t), t \geq 0\right\}$ is referred to as a $Q$-Wiener process if the following conditions are satisfied:

$\left(W_{1}\right) W_{Q}(0)=0$ almost everywhere;

$\left(W_{2}\right) W_{Q}$ has independent increments;

$\left(W_{3}\right)$ the distribution law of increments $W_{Q}(t)-W_{Q}(s)$ is normal with the zero expectation and with covariation operator $(t-s) Q$;

$\left(W_{4}\right) W_{Q}$ has continuous trajectories almost everywhere.

The $Q$-Wiener process thus defined is a generalization of Brownian motion. It is known that, unlike the white noise, the Brownian motion process $\{\beta(t), t \geq 0\}$, where $\beta(t)=\beta(t, \omega)$ and $\omega \in \Omega$ [defined via the validity of the conditions $\left(W_{1}\right)-\left(W_{4}\right)$ for the case in which $\mathbb{H}=\mathbb{R}$ and $Q=I$ ], has continuous trajectories and finite variation. A finite-dimensional Brownian motion is a finite sum of the form $\sum_{i=1}^{n} \beta_{i}(t) e_{i}$, where $e_{i}$ is a basis in $\mathbb{R}^{n}$ and the $\beta_{i}$ are independent Brownian motions. For the passage to the infinite-dimensional case, instead of a divergent series in $\mathbb{H}$, one should consider the regularized sum

$$
W_{Q}(t):=\sum_{i=1}^{\infty} \sigma_{i} \beta_{i}(t) e_{i}, \quad t \geq 0, \quad W_{Q}(t) \in L_{2}(\Omega ; \mathbb{H})
$$

which is an (HI-valued) $Q$-Wiener process. The formal series

$$
\sum_{i=1}^{\infty} \beta_{i}(t) e_{i}=: W(t)
$$

is referred to as a cylindric Wiener process.

\subsection{White Noise in Spaces of Abstract Distributions}

To state the definition of white noise and stochastic convolution in distribution spaces, we introduce some notation. For an arbitrary Banach space $\mathcal{X}$, by $\mathcal{D}^{\prime}(\mathcal{X})$ we denote the space of $\mathcal{X}$-valued distributions on the space $\mathcal{D}$ of test functions, and by $\mathcal{D}_{0}^{\prime}(\mathcal{X})$ we denote the subspace of distributions supported on the positive half-line $[0, \infty)$. Unlike $\mathbb{R}$-valued Schwartz distributions, such distributions are referred to as abstract distributions.

Let $W_{Q}$ be an $\mathbb{H}$-valued $Q$-Wiener process, which, by definition, has trajectories continuous in $t \geq 0$ for almost all $\omega$. We define a $Q$-white noise $\mathbb{W}_{Q}$ in the space $\mathcal{D}_{0}^{\prime}(\mathbb{H})$ as the generalized derivative of a regular distribution (the process $W_{Q}$ extended by zero for $t<0$ ) as follows:

$$
\left\langle\mathbb{W}_{Q}, \theta\right\rangle:=-\int_{0}^{\infty} W_{Q}(t) \theta^{\prime}(t) d t, \quad \theta \in \mathcal{D}
$$

Here the integral is understood as the Bochner integral of a function ranging in the space $L_{2}(\Omega ; \mathbb{H})$ or in the space $\mathbb{H}$ for almost all $\omega$. Note that it follows from the generalization of the integration 
by parts formula $[16$, p. 35] to the case of abstract Itô integrals that the integral on the righthand side in relation (4) coincides with the abstract Itô integral $\int_{0}^{\infty} \theta(t) d W_{Q}(t)$ over the $Q$-Wiener process $W_{Q}$.

We use the convolution of distributions, including a stochastic convolution, in the sense of the following definition.

Definition 3. Let $\mathcal{X}, \mathcal{Y}$, and $\mathcal{Z}$ be Banach spaces such that a bilinear operation $(u, v) \mapsto u v \in \mathcal{Z}$ is defined on $\mathcal{X} \times \mathcal{Y}$. For arbitrary $G \in \mathcal{D}_{0}^{\prime}(\mathcal{X})$ and $F \in \mathcal{D}_{0}^{\prime}(\mathcal{Y})$, the convolution $G * F \in \mathcal{D}_{0}^{\prime}(\mathcal{Z})$ is given by the formula

$$
\langle G * F, \theta\rangle:=\left\langle(g * f)^{(n+m)}, \theta\right\rangle=(-1)^{n+m} \int_{0}^{\infty}(g * f)(t) \theta^{(n+m)}(t) d t, \quad \theta \in \mathcal{D},
$$

where $g: \mathbb{R} \rightarrow \mathcal{X}$ and $f: \mathbb{R} \rightarrow \mathcal{Y}$ are continuous functions such that

$$
\langle G, \theta\rangle=(-1)^{n} \int_{0}^{\infty} g(t) \theta^{(n)}(t) d t, \quad\langle F, \theta\rangle=(-1)^{m} \int_{0}^{\infty} f(t) \theta^{(m)}(t) d t, \quad(g * f)(t):=\int_{0}^{t} g(t-s) f(s) d s .
$$

Note that for the special case in which $G$ is a regular distribution, i.e.,

$$
\langle G, \theta\rangle=\int_{0}^{\infty} G(t) \theta(t) d t
$$

the convolution has the form

$$
\langle G * F, \theta\rangle=\int_{0}^{\infty} G(t)\langle F(\cdot), \theta(t+\cdot)\rangle d t
$$

\subsection{Spaces of Abstract Stochastic Distributions. Singular White Noise}

The theory of stochastic distributions deals with the probability spatial $(\Omega, \mathcal{F}, P)=\left(\mathcal{S}^{\prime}, \mathcal{B}\left(\mathcal{S}^{\prime}\right), \mu\right)$, where $\mathcal{B}\left(\mathcal{S}^{\prime}\right)$ is the Borel $\sigma$-algebra of subsets of the space $\mathcal{S}^{\prime}$ of tempered Schwartz distributions and $\mu$ is the Gaussian measure on $\mathcal{B}\left(\mathcal{S}^{\prime}\right)$, whose existence follows from the Bochner-Minlos theorem (e.g., see [17, p. 12]).

The construction of spaces of abstract stochastic distributions is similar to the construction of the Gelfand triple $\mathcal{S} \subset L_{2}(\mathbb{R}) \subset \mathcal{S}^{\prime}$. Filinkov and Sorensen [18] constructed a triple with middle element the space $\left(L_{2}\right)(\mathbb{H}):=L_{2}\left(\mathcal{S}^{\prime}, \mu ; \mathbb{H}\right)$ of $\mathbb{H}$-valued functions Bochner square integrable with respect to the measure $\mu$ and defined on $\mathcal{S}^{\prime}$,

$$
(\mathcal{S})(\mathbb{H}) \subset \cdots \subset\left(\mathcal{S}_{p}\right)(\mathbb{H}) \subset \cdots \subset\left(L_{2}\right)(\mathbb{H}) \subset \cdots \subset\left(\mathcal{S}_{-p}\right)(\mathbb{H}) \subset \cdots \subset(\mathcal{S})^{*}(\mathbb{H}) .
$$

In the space $\left(L_{2}\right)(\mathbb{H})$, each element $f$ can be expanded in a Fourier series in the Hermite stochastic polynomials $\mathbf{h}_{\alpha}(\omega):=\prod_{i=1}^{\infty} h_{\alpha_{i}}\left(\left\langle\omega, \xi_{i}\right\rangle\right), \omega \in \mathcal{S}^{\prime}$, which form an orthogonal basis in $\left(L_{2}\right)(\mathbb{R})$, with $\mathbb{H}$-valued coefficients $f_{\alpha}$,

$$
f=\sum_{\alpha \in \mathcal{T}} f_{\alpha} \mathbf{h}_{\alpha}, \quad f_{\alpha}=\sum_{j} f_{\alpha, j} e_{j} \in \mathbb{H}, \quad\|f\|_{0, \mathbb{H}}^{2}:=\sum_{\alpha, j} \alpha ! f_{\alpha, j}^{2}=\sum_{\alpha} \alpha !\left\|f_{\alpha}\right\|_{\mathbb{H}}^{2},
$$

where $\mathcal{T}$ is the set of all finite multi-indices $\alpha=\left(\alpha_{1}, \alpha_{2}, \ldots\right)$,

$$
\left\langle\mathbf{h}_{\alpha}, \mathbf{h}_{\beta}\right\rangle=\alpha ! \delta_{\alpha \beta}, \quad \alpha !:=\prod_{i} \alpha_{i} !, \quad \xi_{i}(x)=\pi^{-1 / 4}((i-1) !)^{-1 / 2} e^{-x^{2} / 2} h_{i-1}(x)
$$


are the Hermite functions forming an orthogonal basis in $L_{2}(\mathbb{R})$, and

$$
h_{i}(x)=(-1)^{i} e^{x^{2} / 2} \frac{d^{i}}{d x^{i}} e^{-x^{2} / 2}
$$

are the Hermite polynomials.

The elements of the spaces $\left(\mathcal{S}_{p}\right)(\mathbb{H}), p \in \mathbb{N}$, are defined in terms of the behavior of coefficients of the Fourier series in the basis $\left\{\mathbf{h}_{\alpha}\right\} e_{j}$; namely, $\varphi \in\left(\mathcal{S}_{p}\right)(\mathbb{H})$ if

$$
\varphi=\sum_{\alpha \in \mathcal{T}} \varphi_{\alpha} \mathbf{h}_{\alpha}, \quad \varphi_{\alpha}=\sum_{j} \varphi_{\alpha, j} e_{j} \in \mathbb{H}, \quad\|\varphi\|_{p, \mathbb{H}}^{2}:=\sum_{\alpha, j} \alpha ! \varphi_{\alpha, j}^{2}(2 \mathbb{N})^{2 p \alpha}<\infty
$$

The space $\left(\mathcal{S}_{-p}\right)(\mathbb{H})$ is defined as the dual space of $\left(\mathcal{S}_{p}\right)(\mathbb{H})$; it can be identified with the set of all formal expansions in the system $\left\{\mathbf{h}_{\alpha}\right\} e_{j}$,

$$
\Phi=\sum_{\alpha \in \mathcal{T}} \Phi_{\alpha} \mathbf{h}_{\alpha}, \quad \Phi_{\alpha}=\sum_{j} \Phi_{\alpha, j} e_{j} \in \mathbb{H}, \quad\|\Phi\|_{-p, \mathbb{H}}^{2}:=\sum_{\alpha, j} \alpha ! \Phi_{\alpha, j}^{2}(2 \mathbb{N})^{-2 p \alpha}<\infty .
$$

To construct the desired singular white noise process in these spaces, we use a set

$$
\left\{\beta_{j}(t):=\sum_{i=1}^{\infty} \int_{0}^{t} \xi_{i(n)}(s) d s \mathbf{h}_{\varepsilon_{n(i, j)}}\right\}
$$

of independent Brownian motions, where the $i(n)$ are defined by a bijection such that $n=$ $n(i, j) \geq i j$ and $n(i(n), j(n))=n$; this set was constructed in [7, 18] as a generalization of the expansion of the Brownian motion $\beta \in\left(L_{2}\right)(\mathbb{R})$ in the basis $\left\{\mathbf{h}_{\alpha}\right\}$,

$$
\beta(t, \omega)=\left\langle\omega, \mathbf{1}_{[0, t]}\right\rangle=\left\langle\omega, \sum_{i=1}^{\infty} \int_{0}^{t} \xi_{i}(s) d s \xi_{i}\right\rangle=\sum_{i=1}^{\infty} \int_{0}^{t} \xi_{i}(s) d s \mathbf{h}_{\varepsilon_{i}}, \quad \varepsilon_{i}:=\left(0,0, \ldots,{ }_{i}^{1}, 0, \ldots\right) .
$$

By using the expansions for $\beta_{j}$, we obtain an expansion for the Wiener process $W(t), t \geq 0$,

$$
W(t)=\sum_{j=1}^{\infty} \beta_{j}(t) e_{j}=\sum_{i, j \in \mathbb{N}}\left(\int_{0}^{t} \xi_{i}(s) d s e_{j}\right) \mathbf{h}_{\varepsilon_{n(i, j)}}=\sum_{n=1}^{\infty}\left(\int_{0}^{t} \xi_{i(n)}(s) d s e_{j(n)}\right) \mathbf{h}_{\varepsilon_{n}} .
$$

By virtue of the well-known estimates

$$
\left|\xi_{i}(s)\right|=O\left(i^{-1 / 4}\right), \quad\left\|\int_{0}^{t} \xi_{i}(s) d s e_{j}\right\|_{\mathbb{H}}^{2}=\left|\int_{0}^{t} \xi_{i}(s) d s\right|^{2}=O\left(i^{-3 / 2}\right)
$$

for the Hermite functions, we have

$$
\|W(t)\|_{-1, \mathbb{H}}^{2}=\sum_{i, j \in \mathbb{N}}\left|\int_{0}^{t} \xi_{i}(s) d s\right|^{2}(2 n(i, j))^{-2} \leq C \sum_{i, j \in \mathbb{N}} i^{-7 / 2} j^{-2}<\infty .
$$

Hence it follows that $W(t)=\sum_{j=1}^{\infty} \beta_{j}(t) e_{j} \in\left(\mathcal{S}_{-1}\right)(\mathbb{H})$ for each $t \geq 0$. So much the more, we have

$$
W_{Q}(t)=\sum_{j=1}^{\infty} \sigma_{j} \beta_{j}(t) e_{j} \in\left(\mathcal{S}_{-1}\right)(\mathbb{H}) .
$$

DIFFERENTIAL EQUATIONS $\quad$ Vol. $49 \quad$ No. $4 \quad 2013$ 
Similar estimates imply that the constructed space of generalized $\mathbb{H}$-valued random variables contains the $Q$-white noise $\mathbb{W}_{Q}(t)$ obtained by the formal differentiation of the series for $W_{Q}(t)$ and the singular white noise $\mathbb{W}(t)$ equal to the derivative of $W(t)$,

$$
\mathbb{W}(t)=\sum_{i, j \in \mathbb{N}}\left(\xi_{i}(t) e_{j}\right) \mathbf{h}_{\varepsilon_{n(i, j)}}=\sum_{n \in \mathbb{N}} \mathbb{W}_{\varepsilon_{n}}(t) \mathbf{h}_{\varepsilon_{n}}, \quad \mathbb{W}_{\varepsilon_{n}}(t):=\xi_{i(n)}(t) e_{j(n)}, \quad t \geq 0
$$

They satisfy the relations $\frac{d W_{Q}(t)}{d t}=\mathbb{W}_{Q}(t)$ and $\frac{d W(t)}{d t}=\mathbb{W}(t), t \geq 0$, in $\left(\mathcal{S}_{-1}\right)(\mathbb{H})$.

\section{GENERALIZED SOLUTIONS OF STOCHASTIC CAUCHY PROBLEM WITH WHITE NOISE AND WITH THE GENERATORS OF INTEGRATED SEMIGROUPS}

\subsection{Solution Generalized in $t$ of the Cauchy Problem in Spaces of Abstract Distributions}

Let $A$ be a closed linear operator in $H$, let [dom $A$ ] be the domain of $A$ equipped with the graph norm, let $B \in \mathcal{L}(\mathbb{H} ; H), \zeta \in H$, and let $\mathbb{W}_{Q}$ be the $\mathbb{H}$-valued $Q$-white noise given by relation (4).

By generalizing Fattorini's approach to the case of abstract distributions depending on $\omega$ as a parameter, we define a generalized solution of the Cauchy problem (1) with $\mathbb{W}=\mathbb{W}_{Q} \in \mathcal{D}_{0}^{\prime}(\mathbb{H})$ as a distribution $X \in \mathcal{D}_{0}^{\prime}([\operatorname{dom} A])$ that is a solution of the convolution equation

$$
\langle P * X, \varphi\rangle=\langle\delta \otimes \zeta, \varphi\rangle+\left\langle B \mathbb{W}_{Q}, \varphi\right\rangle, \quad \varphi \in \mathcal{D},
$$

for $\omega$ almost surely, where $P:=\delta^{\prime} \otimes I-\delta \otimes A,\left\langle\delta^{\prime} \otimes I, \varphi\right\rangle:=-\varphi^{\prime}(0) I$, and $\langle\delta \otimes A, \varphi\rangle:=\varphi(0) A$, $\varphi \in \mathcal{D}$.

This equation can also be represented in the more familiar differential form

$$
\left\langle X^{\prime}, \varphi\right\rangle=A\langle X, \varphi\rangle+\langle\delta, \varphi\rangle \zeta+\left\langle B \mathbb{W}_{Q}, \varphi\right\rangle, \quad \varphi \in \mathcal{D},
$$

if we first formally consider $X$ and $\mathbb{W}_{Q}$ as functions, multiply Eq. (1) with the $Q$-white noise by $\varphi \in \mathcal{D}$, integrate the resulting relation from zero to infinity,

$$
\int_{0}^{\infty} X^{\prime}(t) \varphi(t) d t=-\varphi(0) \zeta-\int_{0}^{\infty} X(t) \varphi^{\prime}(t) d t=\int_{0}^{\infty} A X(t) \varphi(t) d t+\int_{0}^{\infty} B \mathbb{W}_{Q}(t) \varphi(t) d t
$$

and then rewrite it in the form of equality of functionals. All terms in the resulting equation (11) are well defined.

A distribution $G \in \mathcal{D}_{0}^{\prime}(\mathcal{L}(H,[\operatorname{dom} A]))$ is called the convolution inverse of $P \in \mathcal{D}_{0}^{\prime}(\mathcal{L}([\operatorname{dom} A], H))$ if $G * P=\delta \otimes I_{[\operatorname{dom} A]}$ and $P * G=\delta \otimes I_{H}$.

By virtue of the properties of the distribution inverse with respect to convolution inverse distribution, it was shown in [9] that the distribution $X$ defined by the formula

$$
\langle X, \varphi\rangle:=\langle G \zeta, \varphi\rangle+\left\langle G * B \mathbb{W}_{Q}, \varphi\right\rangle, \quad \varphi \in \mathcal{D},
$$

is the unique solution of Eq. (10) [and hence of Eq. (11)] that belongs to the space $\mathcal{D}_{0}^{\prime}\left(L_{2}(\Omega,[\operatorname{dom} A])\right)$. The first term on the right-hand side in relation (12) is the deterministic part of the solution depending on the initial conditions, and the second term, given by a generalized stochastic convolution, represents the response of the linear system described by Eq. (10) to the noise $\mathbb{W}_{Q}$.

For the case in which $A$ is the generator of an $n$ times integrated semigroup $V$, the distribution $G$ is the $n$th generalized derivative of $V$, and, in view of relation (4), the representation of the solution (12) acquires the form

$$
\langle X, \varphi\rangle=(-1)^{n}\left[\int_{0}^{\infty} \varphi^{(n)}(t) V(t) \zeta d t-\int_{0}^{\infty} \varphi^{(n+1)}(t) d t \int_{0}^{t} V(t-s) B W_{Q}(s) d s\right] .
$$




\subsection{Solution Generalized in $\omega$ of the Cauchy Problem with the Generator of a Semigroup of the Class $C_{0}$ and with a Singular White Noise in Spaces of Stochastic Distributions}

Consider the Cauchy problem (1) with the singular white noise $\mathbb{W}$ given by relation (9) and with the generator of a semigroup of the class $C_{0}$. Since the singular white noise belongs to the space $\left(\mathcal{S}_{-1}\right)(\mathbb{H})$, for the problem, we have the following assertion [9] on the existence of a solution generalized with respect to $\omega$.

Theorem 1. Let $A$ be the generator of a semigroup $\{U(t), t \geq 0\}$ of the class $C_{0}$ in a Hilbert space $H$. Then, for each $\zeta \in(\operatorname{dom} A) \subset\left(\mathcal{S}_{-1}\right)(H)$, the Cauchy problem (1) with the singular white noise $\mathbb{W}$ has the unique solution

$$
X(t)=U(t) \zeta+\int_{0}^{t} U(t-s) B \mathbb{W}(s) d s \in\left(\mathcal{S}_{-1}\right)(H), \quad t \geq 0 .
$$

Here the domain $(\operatorname{dom} A)$ in $\left(\mathcal{S}_{-1}\right)(H)$ is defined as the set of all $\zeta=\sum_{\alpha} \zeta_{\alpha} \mathbf{h}_{\alpha}$ such that $\zeta_{\alpha} \in$ $\operatorname{dom} A$, and the condition $\sum_{\alpha}\left\|A \zeta_{\alpha}\right\|^{2}(2 \mathbb{N})^{-2 \alpha}<\infty, A \zeta:=\sum_{\alpha} A \zeta_{\alpha} \mathbf{h}_{\alpha}$, is satisfied for $\zeta \in(\operatorname{dom} A)$. The solution of the problem with the singular white noise $\mathbb{W}$ is constructed in the form

$$
X(t)=\sum_{\alpha} X_{\alpha}(t) \mathbf{h}_{\alpha}, \quad t \geq 0
$$

where the corresponding coefficients $X_{\alpha}(t) \in H$ are defined as the solutions of the well-posed Cauchy problems

$$
\begin{aligned}
X_{\varepsilon_{n}}^{\prime}(t) & =A X_{\varepsilon_{n}}(t)+B \mathbb{W}_{\varepsilon_{n}}(t), \quad t \geq 0, \quad X_{\varepsilon_{n}}(0)=\zeta_{\varepsilon_{n}}, \\
X_{\alpha}^{\prime}(t) & =A X_{\alpha}(t), \quad t \geq 0, \quad X_{\alpha}(0)=\zeta_{\alpha} \quad \text { if } \quad \alpha \neq \varepsilon_{n},
\end{aligned}
$$

as follows:

$$
X_{\alpha}(t)= \begin{cases}U(t) \zeta_{\varepsilon_{n}}+\int_{0}^{t} U(t-s) B \mathbb{W}_{\varepsilon_{n}}(s) d s & \text { for } \quad \alpha=\varepsilon_{n} \\ U(t) \zeta_{\alpha} & \text { for } \alpha \neq \varepsilon_{n} .\end{cases}
$$

This, together with definition (9) of a singular white noise, implies the relations

$$
\begin{aligned}
X(t) & =\sum_{\alpha} X_{\alpha}(t) \mathbf{h}_{\alpha} \\
& =\sum_{\alpha} U(t) \zeta_{\alpha}(t) \mathbf{h}_{\alpha}+\sum_{n} \int_{0}^{t} U(t-s) B \mathbb{W}_{\varepsilon_{n}}(s) d s \mathbf{h}_{\varepsilon_{n}}=: U(t) \zeta+\int_{0}^{t} U(t-s) B \mathbb{W}(s) d s .
\end{aligned}
$$

The above-mentioned problems are well-defined in $H$, because $A$ is the generator of a semigroup $\{U(t), t \geq 0\}$ of the class $C_{0}$ and the inhomogeneity $B \mathbb{W}_{\varepsilon_{n}}(t)$ is continuously differentiable with respect to $t \geq 0$ in $H$.

\subsection{SOLUTION OF THE STOCHASTIC CAUCHY PROBLEM GENERALIZED JOINTLY IN $t$ AND $\omega$}

Consider the Cauchy problem (1) with a singular white noise $\mathbb{W}$ and with the generator of an $n$ times integrated semigroup. Let us show that the presence of the white noise and the generator of the integrated semigroup in the considered equation permits one to construct a solution generalized jointly in the time and random variables. Just as in the previous section, we seek the solution of problem (1) as the sum of two terms, the operators of the solution of the corresponding homogeneous Cauchy problem applied to the initial data and the convolution of these operators with the white noise. 
As was mentioned in the introduction, in the case under consideration, essential difficulties are related to the unboundedness of the solution operators $\{U(t), t \geq 0\}$ generated by the operator $A$ and the behavior of the white noise specifying the stochastic inhomogeneity. In the preceding sections, we have shown that, when constructing a generalized solution of the problem with the generator of an integrated semigroup in spaces of abstract distributions, one constructs the distribution of solution operators $U(\cdot)$ and takes a $Q$-white noise $\mathbb{W}_{Q}$ defined in these spaces for the inhomogeneity, while, when constructing a generalized solution in spaces of abstract stochastic distributions, for the inhomogeneity one takes the singular white noise $\mathbb{W}$ defined in these spaces; however, in the latter case, the operator $A$ is assumed to be the generator of a semigroup of the class $C_{0}$.

In our case, the inhomogeneity $\mathbb{W}$ is represented by a singular white noise, and the operator $A$ generates an integrated semigroup. For such $\mathbb{W}$ and $A$, we construct a solution in two stages, which specify the statement of the problem. First, we construct a solution $X_{k}, k \in \mathbb{N}$, generalized with respect to $t$ of the Cauchy problem (1) with stochastic inhomogeneity $\mathbb{W}_{k}$ given by a finite sum of the series defining the singular white noise (9),

$$
\mathbb{W}_{k}(t):=\sum_{i, j=1}^{k}\left(\xi_{i}(t) e_{j}\right) \mathbf{h}_{\varepsilon_{n(i, j)}}, \quad t \geq 0
$$

i.e., we construct a (generalized with respect to $t$ ) solution of Eq. (10) with stochastic inhomogeneity $B \mathbb{W}_{k}$,

$$
P * X_{k}=\delta \otimes \zeta+B \mathbb{W}_{k} .
$$

Then, as $k \rightarrow \infty$, we let $\mathbb{W}_{k}(t)$ tend to $\mathbb{W}(t)$ in the space of stochastic distributions $\left(\mathcal{S}_{-1}\right)(\mathbb{H})$; in the limit, we obtain a solution generalized in the time and random variables.

Theorem 2. Let the operator $A$ be the generator of an $n$ times integrated semigroup $V$. Then, for each $\zeta \in\left(\mathcal{S}_{-1}\right)(H)$, the Cauchy problem (1) with the singular white noise (9) has a unique solution $X \in \mathcal{D}_{0}^{\prime}\left(\left(\mathcal{S}_{-1}\right)(H)\right)$ generalized with respect to $t$ and $\omega$,

$$
\langle\langle X, \theta\rangle, \varphi\rangle=\langle\langle G * \zeta, \theta\rangle, \varphi\rangle+\langle\langle G * B \mathbb{W}, \theta\rangle, \varphi\rangle, \quad \theta \in \mathcal{D}, \quad \varphi \in\left(\mathcal{S}_{1}\right)(H),
$$

where the convolution inverse $G \in \mathcal{D}_{0}^{\prime}(\mathcal{L}(H,[\operatorname{dom} A]))$ of the distribution $P$ is defined as the $n$th derivative of $V$ by the formula $\langle G, \theta\rangle=(-1)^{n}\left\langle V, \theta^{(n)}\right\rangle, \theta \in \mathcal{D}$.

Proof. First, we construct the solution $X_{k} \in \mathcal{D}_{0}^{\prime}(H)$ of problem (15) with the inhomogeneity $B \mathbb{W}_{k}$; then, by letting $k$ tend to infinity, we construct a solution $X$ generalized in $t$ and $\omega$ of problem (1) in the space $\mathcal{D}_{0}^{\prime}\left(\left(\mathcal{S}_{-1}\right)(H)\right)$,

$$
\langle\langle P * X, \theta\rangle \varphi\rangle=\langle\langle\delta \otimes \zeta+B \mathbb{W}, \theta\rangle, \varphi\rangle, \quad \theta \in \mathcal{D}, \quad \varphi \in\left(\mathcal{S}_{1}\right)(H) .
$$

The construction of the solution is carried out in two stages.

The first stage. First, we use the results of Subsection 2.1, where the solution generalized in $t$ of the convolution problem (10) with the generator of the integrated semigroup defining the distribution $G$ inverse to the convolution operator $P$ is constructed by formula (13). By using formula (13), we construct the solution $X_{k}$ of Eq. (15) represented in the form

$$
\left\langle P * X_{k}, \theta\right\rangle=\langle\delta \otimes \zeta, \theta\rangle+\left\langle B \mathbb{W}_{k}, \theta\right\rangle, \quad \theta \in \mathcal{D},
$$

with stochastic inhomogeneity $\mathbb{W}_{k}$ equal to the finite sum (14) for $t \geq 0$ and to zero for $t<0$.

The distribution $\mathbb{W}_{k}$ occurring in Eq. (18), as well as the $Q$-white noise $\mathbb{W}_{Q}$ occurring in Eq. (10), belongs to the space $\mathcal{D}_{0}^{\prime}\left(L_{2}(\Omega ; \mathbb{H})\right)$. Indeed, since each term $\left(\xi_{i}(t) e_{j}\right) \mathbf{h}_{\varepsilon_{n}}$ of the finite sum $\mathbb{W}_{k}(t)$ belongs to the space $\left(L_{2}\right)(\mathbb{H}):=L_{2}\left(\mathcal{S}^{\prime}, \mathcal{B}\left(\mathcal{S}^{\prime}\right), \mu ; \mathbb{H}\right)$, which coincides with $L_{2}(\Omega ; \mathbb{H})$ on $\Omega=\mathcal{S}^{\prime}$, we have $\mathbb{W}_{k}(t) \in L_{2}(\Omega ; \mathbb{H})$. Next, by virtue of the continuity of the functions $\xi_{i}(t), t \geq 0$, this sum is continuous with respect to $t$; consequently, $\mathbb{W}_{k} \in \mathcal{D}_{0}^{\prime}\left(L_{2}(\Omega ; \mathbb{H})\right)$, and moreover, the $\mathbb{W}_{k}$, as well as 
$W_{Q}$, are regular distributions in $\mathcal{D}_{0}^{\prime}\left(L_{2}(\Omega ; \mathbb{H})\right)$. Therefore, to construct a solution with an inhomogeneity that belongs to $\mathcal{D}_{0}^{\prime}\left(L_{2}(\Omega ; \mathbb{H})\right)$, one can use formulas (12) and (13) obtained for the case of $n$ times integrated semigroups and a $Q$-white noise,

$$
\begin{aligned}
\left\langle X_{k}, \theta\right\rangle: & =\langle G \zeta, \theta\rangle+\left\langle G * B \mathbb{W}_{k}, \theta\right\rangle \\
& =(-1)^{n}\left[\int_{0}^{\infty} \theta^{(n)}(t) V(t) \zeta d t+\int_{0}^{\infty} \theta^{(n)}(t) d t \int_{0}^{t} V(t-s) B \mathbb{W}_{k}(s) d s\right] .
\end{aligned}
$$

The constructed solutions $X_{k}$ belong to the space $\mathcal{D}_{0}^{\prime}([\operatorname{dom} A])$ almost surely for all $k$ and $\omega$. In addition, each of these $X_{k}$ belongs to the space $\mathcal{D}_{0}^{\prime}\left(L_{2}(\Omega ; H)\right)$, which, in the case of $\Omega=\mathcal{S}^{\prime}$, coincides with the space $\mathcal{D}_{0}^{\prime}\left(\left(L_{2}\right)(H)\right)$. Consequently, each $X_{k}$ belongs to the space $\mathcal{D}_{0}^{\prime}\left(\left(\mathcal{S}_{-1}\right)(H)\right)$.

The second step. Now let us show that the constructed solutions $X_{k}$ tend as $k \rightarrow \infty$ to the solution $X \in \mathcal{D}_{0}^{\prime}\left(\left(\mathcal{S}_{-1}\right)(H)\right)$ of the Cauchy problem (17) considered in the spaces of distributions with respect to $t$ and $\omega$ and defined by relation (16). To this end, we show that $\left\langle X_{k}, \theta\right\rangle$ is a Cauchy sequence in the complete space $\left(\mathcal{S}_{-1}\right)(H)$ and hence converges to some element $X_{\theta}$ in that space. By virtue of the estimates for coefficients of the expansion of $\left\langle X_{k}, \theta\right\rangle$ in Hermite stochastic polynomials, which, in turn, follow from the boundedness of the functions $\theta \in \mathcal{D}$, the estimates (8), and the boundedness, on any bounded set, of the functions $g(t), t \geq 0$, defined in relation (5) for the stochastic convolution by the distribution $G$ (in the case of an $n$ time integrated semigroup, $\left.G=g^{(n)}, g=V\right)$, we have

$$
\begin{aligned}
\left\|\left\langle\left(X_{k}-X_{k+p}\right), \theta\right\rangle\right\|_{-1, \mathbb{H}}^{2} & =\left\|\sum_{i, j=k+1}^{k+p} \int_{0}^{\infty} \theta^{(m)}(t) d t \int_{0}^{t} V(t-s) \xi_{i}(s) d s e_{j} \mathbf{h}_{\varepsilon_{m(i, j)}}\right\|_{-1, \mathbb{H}}^{2} \\
& \leq C \sum_{i, j=k+1}^{k+p} i^{-1 / 4}(i j)^{-2} \leq \bar{C}(k+1)^{-2} .
\end{aligned}
$$

Therefore, $\left\langle X_{k}, \theta\right\rangle$ is a Cauchy sequence in the space $\left(\mathcal{S}_{-1}\right)(H)$; consequently, its limit element $X_{\theta}$ belongs to $\left(\mathcal{S}_{-1}\right)(H)$. It follows from estimates similar to $(20)$ that $X_{\theta}$ is given by relation (19) with the singular white noise $\mathbb{W}$ instead of $\mathbb{W}_{k}$. In addition, the mapping $X_{\theta}$ is continuous with respect to $\theta \in \mathcal{D}$ and ranges in the space $\left(\mathcal{S}_{-1}\right)(H)$. Consequently, $X_{\theta}=\langle X, \theta\rangle$, where $X \in \mathcal{D}_{0}^{\prime}\left(\left(\mathcal{S}_{-1}\right)(H)\right)$ is given by relation (16).

The resulting solution is unique, because if $X_{1}$ and $X_{2}$ are solutions of problem (10), then $X_{1}-X_{2}$ is a solution of the homogeneous equation with the zero initial condition, which has the unique zero solution; its uniqueness is provided by estimates for the resolvent of an integrated semigroup [6, pp. 44-45]. The proof of the theorem is complete.

In conclusion, consider the Cauchy problem (1) with a singular white noise and with the generators of $R$-semigroups, in particular, semigroups of growth $\alpha \geq 0$. Semigroups of growth $\alpha$ are strongly continuous only for $t>0$, and the generator of a semigroup of growth $\alpha$ is the generator of an integrated semigroup only for $\alpha<1$ and is the generator of an $R$-semigroup in the general case.

\section{SOLUTION OF THE STOCHASTIC CAUCHY PROBLEM WITH WHITE NOISE AND WITH GENERATORS OF $R$-SEMIGROUPS}

In this section, we consider the important special case of $R$-semigroups, a semigroup of growth $\alpha$.

\subsection{Solution of the Cauchy Problem with a Singular White Noise and with the Generators of Semigroups of Growth $\alpha \geq 0$ Generalized for the Variables $t$ and $\omega$}

First, using the results of Section 2, consider the Cauchy problem (1) with a white noise and with the generator of a semigroup of growth $\alpha<1$ as a special case of a problem with the generator of an integrated semigroup; then, in the next section, we consider it as a special case of the generator of an $R$-semigroup for the general case $\alpha \geq 0$. 
A family $\{U(t), t \geq 0\}$ of linear operators bounded in $H$ for each $t>0$ and strongly continuous with respect to $t>0$ is referred to as a semigroup of growth $\alpha$ if the following conditions are satisfied:

$\left(U_{1}\right) U(t+\tau)=U(t) U(\tau), t, \tau>0, U(0)=I$

$\left(U_{\alpha}\right)$ there exist $M, \alpha, \tau>0$ such that $t^{\alpha}\|U(t)\| \leq M, t \in[0, \tau)$.

The operators $U(t), t \geq 0$, are the solution operators of the homogeneous Cauchy problem, but, unlike semigroups of the class $C_{0}$, they are not necessarily bounded in a neighborhood of zero.

If $\alpha<1$, then the generator of a semigroup of growth $\alpha$ is the generator of a once integrated semigroup; consequently, the distributions $X_{k}$ constructed at the first stage of the proof of Theorem 2 are given by the formula corresponding to (19) for $n=1$, and a generalized, with respect to $t$ and $\omega$, solution $X \in \mathcal{D}_{0}^{\prime}\left(\left(\mathcal{S}_{-1}\right)(H)\right)$ of the Cauchy problem with the generator of a semigroup of growth $\alpha<1$ is given by relation (16).

If $\alpha \geq 1$, then the generator of a semigroup of growth $\alpha$ for each $n \in \mathbb{N}$ is not the generator of a semigroup integrated $n$ times; however, as was shown in [19], the generator of a semigroup of growth $\alpha$ is the generator of an $R$-semigroup with operator $R:=\int_{0}^{\infty} t^{[\alpha]} e^{-b t} U(t) d t$, where $b$ exceeds the growth exponent of the semigroup. In the next section, we use this fact and consider the Cauchy problem with the generators of $R$-semigroups. Here, to apply above-obtained results also to the case of $\alpha \geq 1$, we define the Gelfand regularization $\operatorname{reg} U$ of the semigroup $U$ extended by zero for $t<0$ [20, pp. 64-68] by the formula

$$
\langle\operatorname{reg} U, \theta\rangle:=\lim _{\varepsilon \rightarrow 0} \int_{\varepsilon}^{a} U(t)\left[\theta(t)-\theta(0)-\theta^{\prime}(0) t-\cdots-\theta^{([\alpha])}(0) t^{[\alpha]}\right] d t+\int_{a}^{\infty} U(t) \theta(t) d t, \quad a>0 .
$$

It is known that the distribution $\operatorname{reg} U$ coincides with $U$ on $(0, \infty):\langle\operatorname{reg} U, \theta\rangle=\langle U, \theta\rangle, \theta \in \mathcal{D}$, $\operatorname{supp} \theta \in(0, \infty)$.) Then we construct the distribution $G \in \mathcal{D}_{0}^{\prime}(\mathcal{L}(H,[\operatorname{dom} A]))$ via the distribution $\operatorname{reg} U:\langle G, \theta\rangle=\langle\operatorname{reg} U, \theta\rangle, \theta \in \mathcal{D}$, and write out the relation corresponding to (19),

$$
\left\langle X_{k}, \theta\right\rangle=\left[\langle\operatorname{reg} U \zeta, \theta\rangle+\left\langle\operatorname{reg} U * B \mathbb{W}_{k}, \theta\right\rangle\right], \quad \theta \in \mathcal{D} .
$$

Therefore, it follows from the definition of the distribution $G$ and results of Subsection 2.1 that, in the considered cases of semigroups of growth $\alpha$, the solution $X_{k}$ constructed by formula (19) is a solution of problem (18) for $\alpha<1$, and that constructed by formula (21) is a (regularized) solution of problem (18) for $\alpha \geq 1$.

Since, in the construction of a solution generalized with respect to $t$ and $\omega$ and obtained in the case of semigroups of growth $\alpha \geq 1$, the equation $\langle G, \theta\rangle=\langle U, \theta\rangle$ holds only for test functions $\theta$ with supports in $(0, \infty)$ and hence the effect of the initial condition is lost, it follows that the construction of a solution generalized with respect to $\omega$ and the "space" variable of the operator $A$ can be more natural for the generator of a semigroup of growth $\alpha \geq 1$. Let us show how to construct solutions for the Cauchy problem with a singular white noise and with generators of $R$-semigroups (in particular, generators of semigroups of growth $\alpha$ ) with the use of the space of Ivanov generalized functions.

\subsubsection{Solution of the Cauchy Problem with a Singular White Noise and with Generators of R-Semigroups Generalized with Respect to $\omega$ and the "Space" Variable}

Let the operator $A$ occurring in Eq. (1) be the generator of some $R$-semigroup $V=\{V(t)$, $t \in[0, \tau)\}, \tau \leq \infty$. In this case, it follows from the properties of $R$-semigroups and Theorem 1 that, under (quite restrictive) conditions of the boundedness of the operator family $\left\{V(t) R^{-1}\right\}$, for any element $\zeta \in\left(\operatorname{dom} A R^{-1}\right) \subset\left(\mathcal{S}_{-1}\right)(H)$, problem (1) with a singular white noise has a unique solution $X(t) \in\left(\mathcal{S}_{-1}\right)(H), t \in[0, \tau)$, which is given by the relation

$$
X(t)=V(t) R^{-1} \zeta+\int_{0}^{t} V(t-s) R^{-1} B \mathbb{W}(s) d s, \quad t \geq 0 .
$$


Let us show that the solution of problem (1) with the generator of an $R$-semigroup which does not necessarily satisfy the above-mentioned boundedness condition can be constructed in spaces of stochastic distributions $\left(\mathcal{S}_{-1}\right)(\mathcal{H})$ in whose definition the space $\mathcal{H}$ is taken in the form of the spaces of Ivanov generalized functions $H_{-k}$ and $H_{-\infty}$ [5, pp. 150-152] constructed so as to ensure that the operator $R^{-1}$ is bounded in them.

The spaces $H_{-k}$ and $H_{-\infty}$ are introduced as the generalization of spaces of the Schwartz, Sobolev, Zemanyan, and other types in which various differential operators are defined. The action of some unbounded operator $\mathbb{P}=R^{-1}$ (the operator $\mathbb{P}$ in the case under consideration; it is used for the construction of spaces) is defined in Ivanov spaces.

Definition 4. Let $\mathbb{P}$ be a self-adjoint (unbounded) operator in a Hilbert space $H$ with an orthonormal basis of eigenvectors $\left\{e_{k}\right\}$ corresponding to eigenvalues $\left|\mu_{1}\right| \leq\left|\mu_{2}\right| \leq \cdots$ We introduce the Hilbert spaces $H_{k}, k=0,1,2, \ldots$, and the countably Hilbert space $H_{\infty}$ as follows:

$$
H_{k}:=\left\{\varphi \in \operatorname{dom} \mathbb{P}^{k},\|\varphi\|_{k}=\sum_{i=0}^{k}\left\|\mathbb{P}^{i} u\right\|_{H}\right\}, \quad H_{\infty}:=\left\{\varphi \in \bigcap_{k=0}^{\infty} \operatorname{dom} \mathbb{P}^{k}\right\} .
$$

The distribution spaces $H_{-k}, k=0,1,2, \ldots$, and $H_{-\infty}$ are defined as the spaces dual to the spaces $H_{k}$ and $H_{\infty}$, respectively.

An equivalent definition of such spaces can be stated in terms of the behavior of the Fourier coefficients of the elements $\varphi=\sum_{1}^{\infty} \tilde{\varphi}_{j} e_{j}, f=\sum_{1}^{\infty} \tilde{f}_{j} e_{j}$ :

$$
\varphi \in H_{k} \Longleftrightarrow \sum_{j=1}^{\infty}\left|\tilde{\varphi}_{j}\right|^{2}\left|\mu_{j}\right|^{2 k}<\infty, \quad f \in H_{-k} \Longleftrightarrow \sum_{j=1}^{\infty} \frac{\left|\tilde{f}_{j}\right|^{2}}{\left(1+\left|\mu_{j}\right|\right)^{2 k}}<\infty .
$$

The following assertion [5, pp. 153-154] holds for an ill-posed deterministic Cauchy problem with an operator $A$ not generating a semigroup of the class $C_{0}$.

Theorem 3. Let $A$ be a self-adjoint (unbounded) operator in the Hilbert space $H$ that generates a basis of eigenvectors $\left\{e_{j}\right\}$ corresponding to eigenvalues $\lambda_{1} \leq \lambda_{2} \leq \cdots$, and let $\mathbb{P}:=e^{A \tau}, \tau>0$. Then for each $\zeta=\sum_{j \in \mathbb{N}} \zeta_{j} e_{j} \in H_{-k}$, there exists a unique solution of the Cauchy problem $u^{\prime}(t)=$ $A u(t), t \in[0, \tau), u(0)=\zeta$, which can be represented in the form $u(t)=\sum_{j \in \mathbb{N}} e^{\lambda_{j} t} \zeta_{j} e_{j} \in H_{-(k+1)}$ and is stable under changes of $\zeta$ in $H_{-k}$. If $\zeta \in H_{-\infty}$, then $u(t) \in H_{-\infty}$.

One can readily see that the operator $A$ occurring in the assumptions of Theorem 3 is the generator of an $R$-semigroup $\{V(t), t \in[0, \tau)\}$ with the operator $R=e^{-A \tau}$. This, together with Theorems 2 and 3, implies the following assertion on the solvability of the stochastic Cauchy problem (1) with the generator of an $R$-semigroup generalized with respect to the random and "space" variables.

Theorem 4. Let $R$ be a self-adjoint operator in a Hilbert space $H$, let $A$ be the generator of an $R$-semigroup $\{V(t), t \in[0, \tau)\}$ in $H$, let $\left\{\mathbb{W}(t) \in\left(\mathcal{S}_{-1}\right)(\mathbb{H}), t \geq 0\right\}$ be a singular white noise, and let $B \in \mathcal{L}(\mathbb{H}, H)$. Then, for $\mathbb{P}:=R^{-1}$, the function $X(t) \in\left(\mathcal{S}_{-1}\right)\left(H_{-k-1}\right), t \in[0, \tau)$, defined by relation $(22)$ is the unique solution of problem $(1)$ for any $\zeta \in\left(\mathcal{S}_{-1}\right)\left(H_{-k}\right)$, and $X(t) \in\left(\mathcal{S}_{-1}\right)\left(H_{-\infty}\right)$ for any $\zeta \in\left(\mathcal{S}_{-1}\right)\left(H_{-\infty}\right)$.

In conclusion, note that the general principles of regularization in the wide sense $[15,21]$ can be used in all above-considered approaches to the construction of solutions of the Cauchy problem for a stochastically perturbed differential-operator equation. The regularization of the Cauchy problem (1), whose ill-posedness is related to the properties of semigroups generated by the operator $A$ and the properties of the process $\mathbb{W}$ is performed either by using operators correcting the process $\mathbb{W}$ and the semigroup of operators or by an extension of the space of solutions. In this connection, the principle of extension of spaces, which can seem to be quite different, is based on common ideas; it is an extension that permits one to apply some unbounded solution operator arising in the problem. 


\section{ACKNOWLEDGMENTS}

The research was supported by the Program of the Ministry of Education and Science of the Russian Federation (project no. 1.1016.2011) and the Russian Foundation for Basic Research (project no. $\left.10-01-96003_{p}\right)$.

\section{REFERENCES}

1. Da Prato, G. and Zabczyk, J., Stochastic Equations in Infinite Dimensions, Cambridge, 1992.

2. Da Prato, G., Kolmogorov Equations for Stochastic PDIs, Basel, 2004.

3. Filipovic, D., Consistency Problems for Heath-Jarrow-Morton Interest Rate Models, Lecture Notes in Math., vol. 1760, Berlin, 2001.

4. Melnikova, I.V., Filinkov, A.I., and Anufrieva, U.A., Abstract Stochastic Equations. I. Classical and Distributional Solutions, J. Math. Sci., 2002, vol. 111, no. 2, pp. 3430-3465.

5. Ivanov, V.K., Melnikova, I.V., and Filinkov, A.I., Differentsial'no-operatornye uravneniya i nekorrektnye zadachi (Operator-Differential Equations and Ill-Posed Problems), Moscow: Nauka, 1995.

6. Melnikova, I.V. and Filinkov, A., The Cauchy Problem: Three Approaches, Monogr. Surv. Pure Appl. Math., vol. 120, London, 2001.

7. Melnikova, I.V., Filinkov, A.I., and Alshansky, M.A., Abstract Stochastic Equations. II. Solutions in Spaces of Abstract Stochastic Distributions, J. Math. Sci., 2003, vol. 116, no. 5, pp. 3620-3656.

8. Melnikova, I.V. and Filinkov, A.I., Abstract Stochastic Problems with Generators of Regularized Semigroups, J. Commun. Appl. Anal., 2009, vol. 13, no. 2, pp. 195-212.

9. Al'shanskii, M.A. and Melnikova, I.V., Regularized and Generalized Solutions of Infinite-Dimensional Stochastic Problems, Mat. Sb., 2011, no. 11, pp. 3-30.

10. Pilipović, S. and Seleži, D., Structure Theorems for Generalized Random Processes, Acta Math. Hungar., 2007, vol. 117 , no. 3, pp. 251-274.

11. Fattorini, H.O., The Cauchy Problem, in Encyclopedia Math. Appl., Addison-Wesley: Reading, Mass, 1983, vol. 18.

12. Kuo, H.H., Lecture on White Noise Analysis, Soochow J. Math., 1992, vol. 18, no. 3, pp. 299-300.

13. Davies, E.B. and Pang, M.M., The Cauchy Problem and a Generalisation of the Hille-Yosida Theorem, Proc. London Math. Soc., 1987, vol. 55, pp. 181-208.

14. Arendt, W., Vector-Valued Laplace Transforms and Cauchy Problems, Israel J. Math., 1987, vol. 59, pp. 327-352.

15. Melnikova, I.V. and Anufrieva, U.A., Pecularities and Regularization of Ill-Posed Cauchy Problems with Differential Operators, J. Math. Sci., 2008, vol. 148, no. 4, pp. 481-632.

16. Oksendal, B., Stochastic Differential Equations. An Introduction with Applications, 6th ed., Berlin, 2003.

17. Holden, H., Oksendal, B., Uboe, J., and Zhang, T., Stochastic Partial Differential Equations. A Modelling, White Noise Functional Approach, Boston, 1996.

18. Filinkov, A. and Sorensen, J., Differential Equations in Spaces of Abstract Stochastic Distributions, Stoch. Stoch. Rep., 2002, vol. 72, no. 3-4, pp. 129-173.

19. Miyadera, I. and Tanaka, N., Exponentially Bounded C-Semigroups and Generation of Semigroups, Math. Anal. Appl., 1989, vol. 143, no. 2, pp. 358-378.

20. Gel'fand, I.M. and Shilov, G.E., Obobshchennye funktsii. Vyp. 1. Obobshchennye funktsii i operatsii nad nimi (Generalized Functions. I. Generalized Functions and Operations Thereon), Moscow, 1959.

21. Melnikova, I.V., Regularized Solutions to Cauchy Problems Well Posed in the Extended Sense, J. Int. Transf. and Spec. Functions, 2006, vol. 5, pp. 1-7. 